\title{
Molecular Biomarkers of Response to PD-1/ PD-L1 Immune Checkpoint Blockade in Advanced Bladder Cancer
}

\author{
Megan M. Tu ${ }^{\mathrm{a}}$, Terry L. Ng${ }^{\mathrm{b}}$, Florus C. De Jong ${ }^{\mathrm{c}}$, Tahlita C.M. Zuiverloon ${ }^{\mathrm{a}, \mathrm{c}}$, Francesco G.T. \\ Fazzari $^{\mathrm{b}}$ and Dan Theodorescu ${ }^{\mathrm{d}, *}$ \\ ${ }^{a}$ Department of Surgery, University of Colorado Anschutz Medical Campus, Aurora, CO, USA \\ ${ }^{\mathrm{b}}$ Division of Medical Oncology, The Ottawa Hospital Cancer Centre, Ottawa, ON, Canada \\ ${ }^{\mathrm{c}}$ Department of Urology, Erasmus MC Cancer Institute, Rotterdam, The Netherlands \\ ${ }^{\mathrm{d}}$ Cedars-Sinai Samuel Oschin Comprehensive Cancer Institute, Los Angeles, CA, USA
}

Received: 6 February 2019; accepted: 30 April 2019

\begin{abstract}
.
Background: The activity of PD-1/PD-L1 inhibitors in the treatment of advanced bladder cancer (BC) is promising for many patients. However, a subset of patients do not benefit from treatment, thus leading to an effort to better identify predictive molecular biomarkers of response.

Objective: To conduct a systematic review of the literature on predictive molecular biomarkers associated with response to PD-1 and PD-L1 inhibitors in advanced bladder cancer, defined as locally-advanced, unresectable, or metastatic (mBC) disease.

Methods: A search of the literature was performed using Embase (1947 - January 2019), Medline (1946 - January 2019), and EBM Reviews for Cochrane Central Register of Controlled Trials (as of December 2018). Studies examining the association of molecular biomarkers with clinical outcome in BC treated with PD-1 or PD-L1 monotherapy were included. Outcomes of interest were overall survival (OS), cancer-specific survival (CSS), progression-free survival (PFS), duration of response, and objective response rate (ORR).

Results: Using the study search criteria, 899 unique abstract citations were found, of which 834 did not meet the eligibility criteria. Full text of the remaining 65 citations were screened, and 50 studies excluded, including 18 review articles. Eight additional studies from the bibliography of the review papers were included, making a total of 23 studies. Five PD-1 / PD-L1 antibodies have been tested in BC immunohistochemistry (IHC). These studies used different expression scoring criteria and generally had poor ability to discriminate likelihood for response. Overall, the data suggests CD8 ${ }^{+} \mathrm{T}$ cell infiltration is necessary to mediate an antitumor immune response, but other immune cell populations, such as neutrophils may suppress $\mathrm{T}$ cell-mediated immunity and efficacy of PD-1/PD-L1 blockade. An IFN $\gamma$ signature is a promising predictor, but there needs to be consensus on the optimal gene panel composition, and prospective validation. Tumor mutation burden (TMB) is a promising predictor in six studies reporting on 1200 patients, but there is not a consensus on the optimal definition of "high TMB". Detection of T cell receptor (TCR) clonal expansion has only been conducted in small studies and so its predictive value remains inconclusive. Epithelial-mesenchymal transformation (EMT) and transforming growth factor $\beta$ (TGF $\beta$ ) are associated with poor prognosis and possibly intrinsic resistance to PD-1/PD-L1 checkpoint blockade, but more work needs to be done to build upon and confirm the initial findings.
\end{abstract}

\footnotetext{
${ }^{*}$ Correspondence to: Dan Theodorescu, Cedars-Sinai Health System, 8700 Beverly Blvd., OCC Mezz C2002, Los Angeles, CA
} 
Conclusions: Currently no molecular biomarker is sufficiently mature for routine clinical use, while some candidates, or a combination show great promise and need further study.

Keywords: Bladder cancer, biomarkers, immunotherapy, PD-1, PD-L1, atezolizumab, avelumab, durvalumab, nivolumab, pembrolizumab

\section{INTRODUCTION}

Long-term survival in patients with locallyadvanced, unresectable, or metastatic urothelial bladder cancer $(\mathrm{mBC})$ treated with cisplatin-based chemotherapy is poor, with a historical 5-year overall survival rate of $10-15 \%$ [1]. In addition, $50 \%$ of $\mathrm{mBC}$ patients are unfit for cisplatin due to renal function impairment, cardiovascular comorbidities and performance status [2]. The approval of immune checkpoint inhibitors (ICI) in multiple cancer types has reinvigorated excitement for leveraging the immune system to treat cancer. CTLA-4 and PD-1 inhibitors have revolutionized cancer treatment, where previously, chemotherapy was most often the only available treatment for metastatic disease. Exploration of these checkpoint inhibitors has led to their approval in multiple solid tumor types with varying degrees of clinical benefit. In the past two years alone, five ICI treatments have been approved by the FDA in multiple treatment settings [3-7].

Here, we present a systematic review highlighting the current knowledge on molecular biomarkers of response to PD-1 and PD-L1 blockade in the context of advanced bladder cancer.

\section{METHODS}

\section{Literature search}

Electronic search strategies were developed and tested by a medical information specialist (Risa Shorr, The Ottawa Hospital, Ottawa, Ontario, Canada) in consultation with the review team. We searched Ovid MEDLINE (from 1946 to January 2019) and Embase Classic+Embase (from 1947 to January 2019). We also searched EBM Reviews Cochrane Central Register of Controlled Trials up to December 2018. The Cochrane Controlled Register of Trials was first published in 1996, but its composite nature means that it does not have an inception or start date like that of other traditional biomedical databases [8]. Strategies utilized a combination of controlled vocabulary (e.g., "Urinary Bladder Neoplasm") and keywords (bladder cancer or urothelial carcinoma, checkpoint inhibitor). The complete search strategy is outlined in Appendix 1. Vocabulary and syntax were adjusted across databases. Non-human and non-English studies were excluded. The search did not restrict to specific study designs. We also searched for additional references by hand-searching the bibliographies of review papers that met our eligibility criteria. Please refer to Fig. 1 for the Preferred Reporting Items for Systematic Reviews and Meta-Analyses (PRISMA) [9] flowchart. PRISMA is an evidence-based minimum set of items for reporting in systematic reviews and meta-analyses.

\section{Study selection criteria and data collection}

Selection criteria for randomized studies were characterized according to the PopulationIntervention-Comparators-Outcomes-Study design (PICOS) framework [10]. Non-randomized studies utilized a similar framework. The study population of interest were patients diagnosed with advanced $\mathrm{BC}$, defined by the authors as locally advanced (on the TNM staging system, T4b and any N; or any T and N2-3), unresectable (unable, unfit or unwilling to undergo a cystectomy) or metastatic (M1, stage IV: spread to a distant site outside of the bladder; non-regional lymph nodes or visceral metastases) urothelial bladder cancer, who received anti-PD-1 or anti-PD-L1 monotherapy. Studies that included $\mathrm{mBC}$ only, as well as studies that included them as a subgroup of the study population were eligible. We only included studies that investigated the predictive value of molecular biomarkers, defined as a measurable marker in blood or tissue that has the potential to predict response and clinical outcome. Outcomes of interest included overall survival (OS), progression-free survival (PFS), time-to-progression (TTP), duration of response (DoR), objective response rate (ORR), as well as derivatives of these clinical endpoints.

In an initial screen, MMT and TLN independently evaluated all abstracts identified by the literature search. A second assessment of full-text articles was performed by MMT, TLN, TCMZ, FCdJ, and 


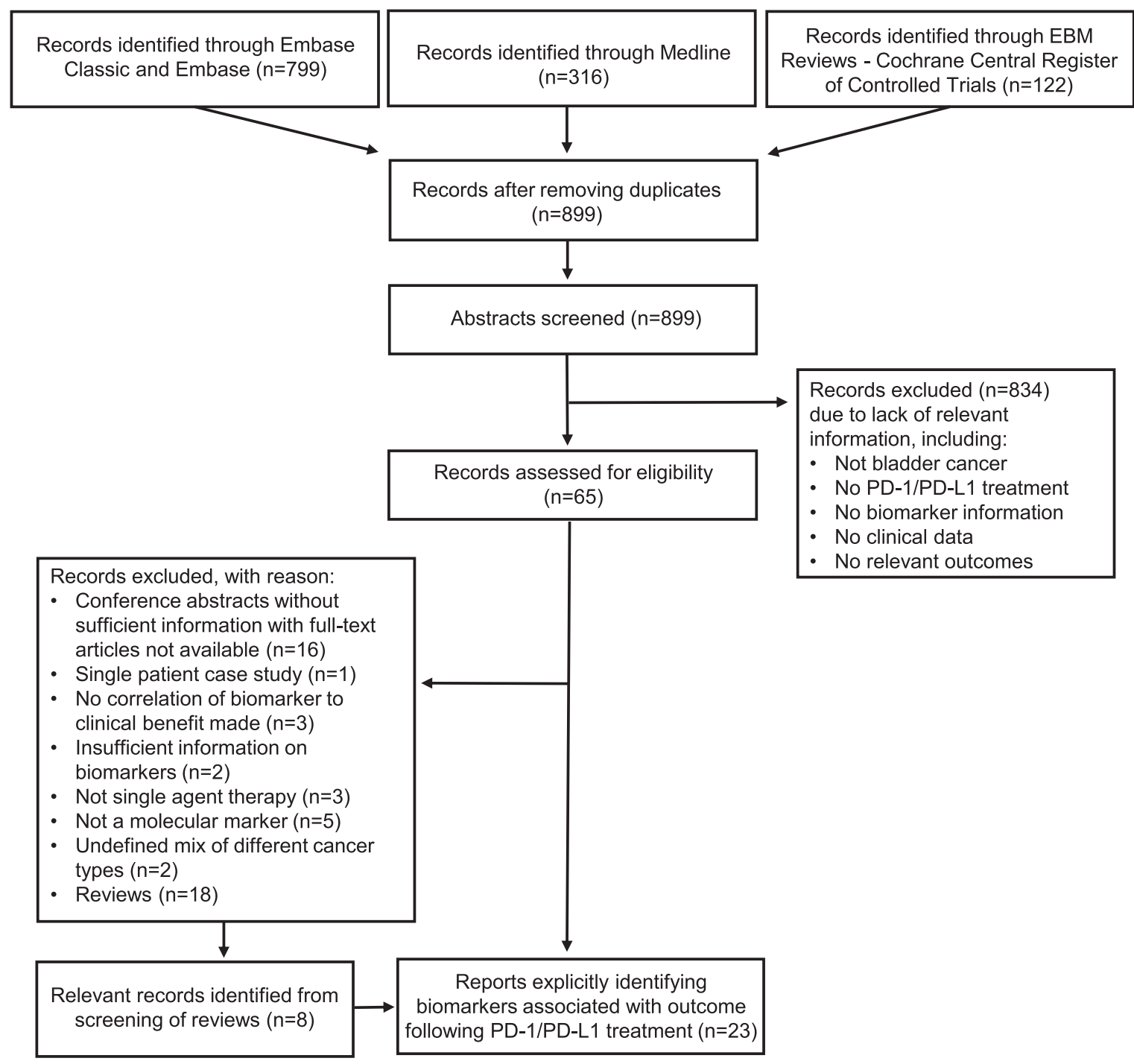

Fig. 1. PRISMA flowchart outlining the selection criteria for the systematic review.

FGTF. Reasons for study exclusion were recorded and reported in Fig. 1. The summary of the literature selection process according to the PRISMA guidelines is reported in Fig. 1. We collected data from included studies about study design (randomized clinical trial, prospective or retrospective cohort study, case series), sample size, name of anti-PD-1 or anti-PD-L1 antibody, type of molecular biomarker examined, method of marker measurement, and clinical endpoints reported. Collection of study characteristics and outcomes was performed by MMT and TLN using a structured form. MMT, TLN, TCMZ, FCdJ, and FGTF performed primary data collection. MMT verified all items extracted by other reviewers.
Primary data extracted by MMT was independently verified by TLN.

\section{RESULTS}

\section{PD-L1 expression}

\section{Atezolizumab}

The earliest study to demonstrate efficacy of PDL1 blockade in $\mathrm{mBC}$ was the phase 1 expansion study examining the efficacy of atezolizumab in a cohort of 205 cisplatin-pretreated $\mathrm{mBC}$ patients [3]. The cohort with PD-L1 positive tumor-infiltrating immune cells (IC) had particularly high response 
Table 1

Complementary diagnostic assays for PD-L1 expression used as a molecular biomarker in clinical trials

\begin{tabular}{|c|c|c|c|c|c|}
\hline $\begin{array}{l}\text { PD-L1 } \\
\text { Antibody }\end{array}$ & Manufacturer & $\begin{array}{l}\text { ICI used in } \\
\text { the study }\end{array}$ & Scoring method & Scoring cutoffs & Ref. \\
\hline \multirow[t]{2}{*}{$22 \mathrm{C} 3$} & Dako & Pembrolizumab & $\begin{array}{l}\text { PD-L1-expressing TC and } \\
\text { IC, relative to total TC }\end{array}$ & $\begin{aligned} & \text { Positive }: \geq 10 \% \\
& \text { Negative }:<10 \%\end{aligned}$ & {$[16,18]$} \\
\hline & & & $\begin{array}{l}\text { PD-L1 detected on TC } \text { or in } \\
\text { tumor stroma }\end{array}$ & Positive: $\geq 1 \%$ Negative: $<1 \%$ & [5] \\
\hline \multirow[t]{2}{*}{$28-8$} & Dako & Nivolumab & PD-L1 on TC & Positive: $\geq 1 \%$ Negative $:<1 \%$ & {$[4,36,47]$} \\
\hline & & & & Positive: $\geq 5 \%$ Negative $:<5 \%$ & {$[4,36]$} \\
\hline \multirow[t]{2}{*}{ SP263 } & Roche & Durvalumab & PD-L1 on TC or IC & High: $\geq 25 \%$ Low $:<25 \%$ & {$[6,41,66]$} \\
\hline & & & PD-L1 on TC and/or IC & $\begin{array}{l}\text { High: } \geq 25 \% \text { of either TC or } \\
\text { IC Low or negative: }<25 \% \\
\text { of both TC and IC }\end{array}$ & {$[27]$} \\
\hline \multirow[t]{2}{*}{ SP142 } & Roche & Durvalumab & PD-L1 on TC & Positive: $\geq 1 \%$ Negative $:<1 \%$ & [29] \\
\hline & & Atezolizumab & PD-L1 on IC & $\begin{array}{l}\text { IC } 0:<1 \% \text { IC } 1: \geq 1 \% \text { to }<5 \%) \\
\text { IC } 2 / 3: \geq 5 \%\end{array}$ & {$[3,11,12,14,45]$} \\
\hline \multirow[t]{2}{*}{$73-10$} & Merck KGaA & Avelumab & PD-L1 on TC & $\begin{array}{l}\text { Positive: } \geq 1 \% \text { (any } \\
\quad \text { intensity), } \geq 5 \% \text { (any } \\
\text { intensity), or } \geq 25 \% \\
(\geq 2+\text { staining intensity) }\end{array}$ & [7] \\
\hline & & & PD-L1 on IC & Positive $: \geq 10 \%$ & \\
\hline
\end{tabular}

Abbreviations: ICI, immune checkpoint inhibitor; TC, tumor cell; IC, immune cell.

rates, with many patients having a durable response [3]. Moreover, elderly mBC patients often present with impaired renal function, which is a major limiting issue for chemotherapeutic treatment options in $\mathrm{mBC}$. Atezolizumab's more favorable toxicity profile, including less renal toxicity, led to further development of atezolizumab in both the cisplatinpretreated and cisplatin-ineligible population [11, 12]. In a single-arm phase 2 study of $310 \mathrm{mBC}$ patients who progressed after previous platinumbased chemotherapy, assuming a historical control ORR of $10 \%$, atezolizumab demonstrated a significantly improved RECISTv1.1[13] ORR in the overall study population (15\% [95\% CI: 11-20], $p=0.0058$ ), in the PD-L1 immune cell (IC) $1 / 2 / 3$ group (PD-L1 expression $\geq 1 \%) \quad(18 \% \quad[95 \% \mathrm{CI}$ : 13-24], $p=0.0004$ ), and in the IC $2 / 3$ group (PD-L1 expression $\geq 5 \%)(27 \%$ [95\% CI: 19-37], $p<0.0001)$ (Table 1, complementary diagnostic PD-L1 assay SP142) [11]. The single-arm phase 2 study also showed that tumor mutational burden (TMB) and TCGA luminal cluster 2 were associated with an increased response rate [11].

Table 1 highlights the different antibodies currently used in the clinic to measure PD-L1 expression. It should be noted that the antibodies currently used vary based on the immune checkpoint inhibitor of the study, as well as having different thresholds for expression level classification.

In another single-arm phase 2 study of 119 untreated cisplatin-ineligible $\mathrm{mBC}$ patients, ate- zolizumab demonstrated an improvement in ORR [12]. However, there was no difference in ORR, PFS, or OS based on stratification of PD-L1 expression on ICs. In May 2016, the FDA granted accelerated approval to atezolizumab based on promising efficacy data in single arm phase 2 studies for both cisplatinpretreated and cisplatin-ineligible $\mathrm{mBC}$ patients [11, 12].

Although these two studies led to the accelerated approval of atezolizumab, a phase 3 randomized control trial of 931 platinum-pretreated $\mathrm{mBC}$ patients randomized to atezolizumab or second-line chemotherapy (vinflunine, paclitaxel, or docetaxel) did not demonstrate improved overall survival in the IC 2/3 subgroup, which was their pre-planned primary endpoint based on previous studies [14]. There was no difference in the ORR. However, there was a numerical difference in duration of response (15.0 months versus 8.3 months) favoring atezolizumab. Further analyses from this study confirmed the lack of difference in OS regardless of PD-L1 subgroup (both IC $2 / 3$ and IC $1 / 2 / 3$ ). Although there was a significant improvement in OS favoring atezolizumab over chemotherapy in the intention-to-treat (ITT) population ( $n=931), \mathrm{HR}=0.85$ (95\% CI: 0.73 to 0.99$)$, a median OS (mOS) difference of 0.6 months $(8.6$ months vs. 8.0 months) was not clinically meaningful. However, further assessment of the high TMB subgroup $(n=274)$ did show a meaningful difference in mOS (11.3 versus 8.3 months, HR 0.68 [95\% CI: 0.51 to 0.80$]$ ) favoring atezolizumab [15]. 
Table 2

Clinical benefit of biomarkers to PD-1/PD-L1 therapy

\begin{tabular}{|c|c|c|c|}
\hline Biomarker & $\begin{array}{c}\text { Biomarker Predictive } \\
\text { Value }\end{array}$ & $\begin{array}{l}\text { Total sample size } \\
(n=)\end{array}$ & Reference \\
\hline \multirow{6}{*}{ PD-L1 } & \multirow{3}{*}{+} & Atezolizumab: 515 & {$[3,11]$} \\
\hline & & Durvalumab: 191 & {$[6,27]$} \\
\hline & & Avelumab: 293 & {$[7,28]$} \\
\hline & \multirow{3}{*}{-} & Atezolizumab: 586 & {$[12,14]$} \\
\hline & & Pembrolizumab: 384 & {$[16,18,29]$} \\
\hline & & Nivolumab: 351 & {$[4,36]$} \\
\hline TCR clonal expansion & + & 44 & {$[29,46]$} \\
\hline $\mathrm{CD}^{+} \mathrm{T}$ cell infiltration & + & 124 & {$[29,30]$} \\
\hline \multirow{2}{*}{ Tumor mutation burden } & + & 1107 & {$[11,12,14,29,45,47]$} \\
\hline & - & 29 & {$[46]$} \\
\hline $\mathrm{IFN \gamma}$ & + & 301 & {$[36,40,41]$} \\
\hline
\end{tabular}

Abbreviations: TCR, T cell receptor; IFN, interferon; +, predictive of clinical benefit; -, not predictive of clinical benefit.

Overall, atezolizumab showed promising early results $[3,11,12]$, which was unfortunately not validated in the phase 3 trial [14]. Based on the current data, PD-L1 expression using the SP142 assay cannot be used to predict clinical benefit from atezolizumab (Table 2).

\section{Pembrolizumab}

The KEYNOTE-012 study $(n=33)$ first established safety and efficacy of pembrolizumab in $\mathrm{mBC}$ [5]. PD-L1 expression was examined on both tumor and stroma and found ORR to be higher in the PDL1-negative tumor cell (TC) subgroup compared to the PD-L1-positive TC subgroup (27\% vs. $14 \%$ ) (Table 1, complementary diagnostic assay PD-L1 clone 22C3). In contrast, patients with detectable PD-L1 expression on both TC and stroma had higher ORR than those that were PD-L1 negative in both TC and stroma (ORR 24\% versus 0\%) [5]. Two studies reported on the efficacy of pembrolizumab in patients that recurred or progressed after platinum-based chemotherapy, and in patients that were ineligible for platinum-based treatment, respectively. In KEYNOTE-045, a phase 3 randomized study of 542 platinum-pretreated $\mathrm{mBC}$ patients, 270 patients randomized to pembrolizumab had a significantly improved OS compared to single agent chemotherapy [16]. A two-year follow-up showed an OS benefit with pembrolizumab in all PD-L1 subgroups (HR: Combined Positive Score [CPS] $<1 \%, 0.82 ; \quad \mathrm{CPS} \geq 1 \%, 0.58 ; \mathrm{CPS}<10 \%$, 0.75 ; CPS $\geq 10 \%, 0.56$ ) (Table 1, Complementary diagnostic PD-L1 assay Dako 22C3) [17]. For firstline, cisplatin-ineligible patients, KEYNOTE-052, a single-arm phase 2 trial of $370 \mathrm{mBC}$ patients had an ORR of 24\% in the overall cohort and an ORR of $38 \%$ in the subgroup with a PD-L1 expression $\geq 10 \%$ [18]. Longer follow-up on this study confirmed an ORR of $29 \%$ in the ITT group and ORR $47 \%$ in the PD-L1 CPS $\geq 10 \%$ group. Sensitivity and specificity testing of the first 100 patients who had tissue for analysis showed that, PD-L1 CPS had a ROC AUC $0.58, p=0.111(n=96)$. The variability of the available study data suggests that PD-L1 immunohistochemistry (IHC) does not have the ability to discern responders from non-responders. The results of KEYNOTE-045 and KEYNOTE-052 support the use of pembrolizumab in $\mathrm{mBC}$ in different clinical scenarios.

From the IMvigor130 (atezolizumab) and KEYNOTE-361 (pembrolizumab) studies, both randomized studies that contained a chemotherapy monotherapy arm and a PD-1 (pembrolizumab) or PD-L1 (atezolizumab) inhibitor monotherapy arm, the Data Monitoring Committee (DMC) noted that in subgroups with low PD-L1 expression, patients receiving pembrolizumab or atezolizumab monotherapy had worse overall survival compared to patients receiving platinum-based chemotherapy based on the preliminary analysis [19-21]. As such, both the KEYNOTE-361 and the IMvigor130 trials have stopped enrolling patients with PD-L1-low status to the monotherapy arms, and the FDA now requires PD-L1 testing for patients receiving first-line pembrolizumab or atezolizumab unless they are not eligible to receive any platinum-based chemotherapy [19]. These results highlight the need for ICI use to be anchored by careful selection of 
patients that will most likely benefit from treatment. This is compounded by the financial costs and clinical toxicity associated with these agents [22-25].

\section{Nivolumab}

A multi-arm phase 1/2 study (CheckMate-032) of $86 \mathrm{mBC}$ patients with recurrent metastatic urothelial carcinoma reported an investigator-assessed ORR of 24.4\% (95\% CI: 15.3-35.4) [26]. ORR, 1- and 2-year PFS and OS rates were similar between PD-L1 $<1 \%$ and $>1 \%$ subsets (Table 2 , complementary diagnostic PD-L1 assay, Dako 28-8) [26]. In a subsequent single-arm phase 2 study of 265 cisplatin-pretreated patients, ORR was achieved in $19.6 \%$ (95\% CI 15.0 to 24.9$)$ in the overall cohort $(N=265), 28.4 \%$ (95\% CI: 18.9 to 39.5$)$ in the PD-L1 $\geq 5 \%$ cohort $(N=81)$, $23.8 \%$ (95\% CI: 16.5 to $32.3 \%$ ) in the PD-L1 $\geq 1 \%$ cohort $(N=81)$, and $16.1 \%$ (95\% CI: 10.5 to 23.1$)$ in the PD-L $1<1 \%$ cohort $(N=143)$. In these earlier phase studies, higher PD-L1 expression seemed to enrich for responders, but a significant proportion of patients still responded despite being PD-L1 negative.

\section{Durvalumab}

In the expansion cohort of a phase $1 / 2$ openlabel study consisting of $61 \mathrm{mBC}$ patients, most $(93.4 \%)$ of whom received one or more prior therapies for metastatic disease, the ORR was $31 \%$ (95\% CI: 17.6 to 47.1 ) in 42 response-evaluable patients, $46.4 \%$ (95\% CI: 27.5 to 66.1) in the PDL1 positive subgroup, and $0 \%$ (95\% CI: 0 to 23.2 ) in the PD-L1 negative subgroup (Table 1, complementary diagnostic PD-L1 assay SP263). Twelve of 13 responders have ongoing response (range: 4.1 to 49.3 weeks, median not reached) [6]. Updated results from the same phase 1/2 open-label study, consisting of 191 platinum-pretreated or platinumineligible $\mathrm{mBC}$ patients demonstrated an ORR of $17.8 \%$ (95\% CI: 12.7 to 24 ) in the overall cohort, ORR of $27.6 \%$ (95\% CI: 19 to 37.5 ) in the PD-L1 positive ( $n=27$, PD-L1 $\geq 25 \%$ TC or IC), and ORR of 5.15 (95\% CI: 1.4 to 12.5 ) in the PD-L1 low or negative cohort (PD-L1 $<25 \%$ in both TC and IC) [27]. In a single phase $1 / 2$ study with a relatively large expansion cohort, PD-L1 expression seems a promising predictor of response, with the PD-L1 low or negative cohort having low response rates. Longer term follow-up and larger confirmatory studies need to look at the value of this PD-L1 assay for PFS and OS.

\section{Avelumab}

A phase $1 \mathrm{~b}$ cohort of 44 treatment-refractory $\mathrm{mBC}$ patients reported an independently assessed ORR $18.2 \%$ (95\% CI: 8.2 to $32.7,5$ complete response and 3 partial responses). Seven of 8 responding patients had $\mathrm{PD}-\mathrm{L} 1$ positive (PD-L1 TC $\geq 5 \%$ ) tumors (Table 1, complementary diagnostic PD-L1 assay 73-10) [7]. A pooled analysis combining two phase 1 study cohorts $(n=249)$ comprising platinumpretreated patients and platinum-ineligible patients reported an ORR 24\% in the PD-L1 positive subgroup and an ORR $13 \%$ in the PD-L1 negative subgroup [28]. With this relatively small mixed population of platinum-pretreated and platinum-ineligible patients, determining the value of PD-L1 score conclusively is challenging.

In conclusion, PD-1 and PD-L1 ICIs have become commonplace in the treatment of many solid tumor types including bladder cancer. Clearly, only a subset of mBC patients benefit from these agents significantly. The use of PD-L1 expression to predict response is limited by the heterogeneity of PD-L1 expression over time and geography, but also that there are different diagnostic platforms and criteria for scoring PD-L1 expression (Table 1). Examining the present literature, PD-L1 testing seems to have the greatest discriminatory ability of responders and nonresponders to durvalumab. However, a larger phase 3 study for durvalumab has yet to confirm these trends.

\section{$\mathrm{CD8}^{+} \mathrm{T}$ CELL INFILTRATION}

Response to immunotherapy is dependent on $\mathrm{T}$ cells invading the tumor and inducing an effective immune reaction. Therefore, studies have focused on tumor infiltrating lymphocytes, such as $\mathrm{CD} 8^{+} \mathrm{T}$ cells, as a biomarker of response to therapy. In a study of 18 patients treated with pembrolizumab, durvalumab or atezolizumab, $\mathrm{CD} 8^{+} \mathrm{T}$ cell infiltration assessed by IHC significantly correlated with cancer-specific survival (CSS) $(p=0.013)$ [29]. Of the 212 patients from a phase 2 trial of nivolumab in $\mathrm{mBC}$ (CheckMate275), 106 patient samples were analyzed for $\mathrm{CD}^{+}$ $\mathrm{T}$ cells in the tumor by IHC, and which showed that high CD8 expression is associated with longer PFS $(p=0.0003)$ and OS $(p=0.01)$ [30].

The ratios of other immune cell subsets relative to $\mathrm{CD}^{+} \mathrm{T}$ cells have also been used to better understand the immune response in the tumor microenvironment [31]. Inflammation is an important marker of immunotherapy treatment outcome in cancer. Neu- 
trophils have been associated with poor prognosis since they can suppress cytotoxic $\mathrm{T}$ cell activity [32, 33]. In a phase $1 / 2$ open-label expansion study of durvalumab in patients with locally-advanced and $\mathrm{mBC}$, lower neutrophil-to-lymphocyte ratio at baseline was associated with increased tumor response rate as well as prolonged survival [34].

$\mathrm{CD}^{+} \mathrm{T}$ cell infiltration into the tumor microenvironment is necessary, but not sufficient to predict anti-cancer response. This suggests that response to immunotherapy is not dependent on only one cell subset, but more so on the proportion of the different immune cell subsets, specifically a balance between the anti-tumor, such as $\mathrm{CD} 8^{+} \mathrm{T}$ cells, amongst the other cells in the tumor microenvironment.

\section{Interferon- $\gamma(I F N \gamma)$ signature}

IFN $\gamma$ is a requisite mediator of inflammation, with a critical role in the recruitment of $\mathrm{T}$ cells and additional cellular mediators of the immune response [35]. From a phase 2 trial of nivolumab in metastatic UC (CheckMate-275), a 25-gene IFN $\gamma$ and a 12-gene chemokine expression profile was generated from tumor tissue samples of 177 patients [36]. Based on the 25-gene IFN $\gamma$ signature, a high IFN $\gamma$ signature was associated with response to nivolumab $(p=0.0003)$. The authors also noted that the 12-gene chemokine signature was highly enriched in tumors from nivolumab responders, though the data was not shown [35]. Overall, individuals who responded to nivolumab were enriched for anti-tumor-associated immune biomarkers such as CXCL9, CXCL10, and CD8, which were part of the multi-gene signature. CXCL9 and CXCL10 are ligands for the CXCR3 and have been shown to be mediators of $\mathrm{CD}^{+} \mathrm{T}$ cell response [37-39].

In a retrospective study of patients treated with durvalumab, RNA sequencing of 97 non-small cell lung cancer (NSCLC) patient tumors was undertaken as part of a discovery set, which identified a four-gene IFN $\gamma$ signature [40]. The four-gene panel consisted of IFN $\gamma$, CD274, LAG3 and CXCL9. The signature was validated in 62 locally-advanced or $\mathrm{mBC}$ patients, which showed a 3.5-fold higher ORR in patients with the IFN $\gamma$ signature compared with those who were absent of the biomarker. Utilizing the same four-gene IFN $\gamma$ signature in another study, patients treated with durvalumab within the top third of IFN $\gamma$ signature tumor expression were scored as positive [41]. These patients had increased ORR ( $45 \%$ in IFN $\gamma$ signature positive cohort vs $16 \%$ in IFN $\gamma$ signature negative), as well as improved PFS $(p=0.005)$ and OS $(p=0.016)$ compared to the IFN $\gamma$ signature negative cohort [41].

As part of the KEYNOTE-012 study, a phase $1 \mathrm{~b}$ study of pembrolizumab in multiple solid tumor types, RNA sequencing was undertaken in an initial discovery set of 19 melanoma patient tumor samples followed by validation in 9 other solid tumor types comprising 220 patients, of which 25 were bladder cancer patient samples [42]. From this, a 10-gene IFN $\gamma$ signature and an expanded 28-gene immune signature was derived. This study did not use these gene signatures to determine if there was correlation with clinical benefit in bladder cancer, since it was used as part of the discovery dataset. The IFN $\gamma$ and immune signatures were used in the head and neck squamous cell carcinoma (HNSCC) $(n=43)$ and gastric cancer cohort $(n=33)$, with results showing that a higher IFN $\gamma$ signature score was correlated with increased PFS ( $p<0.001$ and $p=0.032$, respectively), compared to those with a low IFN $\gamma$ signature score. While this study did not perform validation studies with bladder cancer data, results were confirmed in two other solid tumor types. IFN $\gamma$ signature is a promising predictive molecular biomarker that may complement other biomarkers. Overall, studies were in heterogeneous populations, used different IFN $\gamma$ associated genes measured by different techniques, and findings have not been validated in independent prospective studies.

\section{Tumor Mutation Burden (TMB)}

$\mathrm{BC}$ has one of the highest mutation rates after lung carcinoma and melanoma [43]. TMB has been strongly correlated with clinical benefit in other solid tumors, and therefore is of great interest in BC as well [44]. Whole exome sequencing was performed on 250 patient samples from the IMvigor210 study; TMB and tumor neoantigen burden correlated with response to atezolizumab $\left(p=6.9 \times 10^{-7}\right.$ and $p=2.7 \times 10^{-9}$, respectively) [45]. TMB was also positively associated with $\mathrm{OS}\left(p=2.0 \times 10^{-5}\right)$ [45]. The pathways most significantly associated with TMB included cell cycle, DNA replication, and DNA damage response (DDR) [45]. Tumors with at least one mutation in DDR or cell cycle regulator genes had a significantly higher TMB and higher response rates to treatment $(p=0.0117)$ [45].

In contrast, another analysis also using data from the IMvigor210 study, but looking at a smaller group of just 29 patients saw no correlations with respect to $\mathrm{TMB}$, or expressed or predicted neoantigen load 
with durable clinical benefit [46]. The differences between these two studies highlights the variability amongst samples within a given trial, and the difference in interpretations given a specific sample size. The authors of the 29-patient study went on to pursue an alternate analysis of their patient data to examine time-varying association between mutation load and PFS, to determine if mutation load had a differential association with three months post-treatment versus more than three months posttreatment [46]. This alternate analysis found evidence of time-varying effects of somatic mutation load on PFS $(p=0.044)[46]$. In comparing TMB within the first 3 months post-treatment versus more than 3 months post-treatment, there was a stronger association of TMB with PFS at more than 3 months after treatment, compared to that in the first 3 months [46]. This suggests that the association between mutation load as a predictor of response is better over time.

In a phase 2 study looking at atezolizumab in firstline treatment in 119 cisplatin-ineligible patients with locally-advanced and $\mathrm{mBC}$, TMB was found to be higher in responders than non-responders, and was consistent across TCGA subtypes and PD-L1 expression groups [12]. Higher TMB was associated with better OS. The level of TMB had a direct relationship with prolonged survival, where patients within the top $25 \%$ of mutation load had longer survival than those in the lower 75\% [12]. In another study of atezolizumab, looking at 310 patients with locallyadvanced and metastatic urothelial carcinoma who had progressed after platinum-based chemotherapy, a correlation between higher TMB and response to atezolizumab was also observed [11].

The impact of TMB on nivolumab efficacy in second-line BC patients was examined in CheckMate 275 , a phase 2 study in 139 patients with analyzable samples [47]. High TMB was associated with higher ORR [TMB high, medium, low $(31.9 \%, 17.4 \%$, $10.9 \%), p=0.002$ ] and PFS [TMB high, medium, low (3.02, 1.87, 1.91 months), $p=0.005]$, but not a significantly different OS [TMB high, medium, low (11.63, 9.66, 5.72 months), $p=0.067]$. TMB was defined in this study as the total number of somatic missense mutations per tumor, and was evaluated as a continuous variable as high, middle/medium or low tertiles [47]. Lastly, whole exome sequencing of 14 bladder cancer patients treated with pembrolizumab, atezolizumab, or durvalumab examined the number of nonsynonymous mutations [29]. The study showed that a high TMB and neoantigen burden correlated with CSS ( $p=0.034$ and $p=0.047$, respectively). In this study, high was defined as greater than or equal to the median value.

Although pembrolizumab has been approved to treat all unresectable or metastatic solid tumors that are microsatellite instability-high (MSI-H) or mismatch repair deficient (dMMR), the occurrence of MSI-H or dMMR is rare. The presence of mismatch repair deficiency may lead to a higher mutation load or TMB. One study performed massively-parallel sequencing and interrogation on 447 tumors from 424 urothelial cancers of different stages, and found only 13 out of $424(3 \%)$ tumors were MSI-H [48]. Patients with MSI-H tumors $(n=13)$ had a median mutation count of $52(36.5-73.5)$ versus $8(5-13)$ in nondMMR patients $(n=410)(p<0.01)$. All five of the 13 MSI-H patients that received immune checkpoint blockade (drug not specified) for metastatic disease achieved near complete or complete responses. All 13 dMMR patients were still alive compared to 125 out of 410 non-dMMR patients at 27 months of follow-up ( $p=0.014$ ) [48]. Based on these data, high TMB may be a good discriminator of responders and non-responders, but questions remain regarding the optimal definition of high TMB, the panel of interrogated genes, and the accuracy of the techniques and technology. Despite being rare, MSI-H / dMMR status should be a strong predictor of clinical benefit from PD-1 blockade in those who have it.

\section{T cell receptor (TCR) clonality}

$\mathrm{T}$ cell receptors (TCR) recognize antigens presented by the major histocompatibility complex (MHC). The immune system is marked by TCR diversity, which is indicative of how many different antigens the $\mathrm{T}$ cell can recognize and respond to. The clonality of the TCR, indicated by a higher proportion of one subset, suggests a clonal expansion of $\mathrm{T}$ cells in response to a specific stimulus. TCR clonality can be detected by sequencing of the CDR3 region of the $\mathrm{T}$ cell and high prevalence of a sequence is indicative of $\mathrm{T}$ cell expansion in response to a stimulus, for instance a tumor antigen. Higher TCR clonality has been shown to be associated with response to immune checkpoint blockade in melanoma, breast and prostate cancer [49-52]. In an analysis of 15 patients with urothelial carcinoma treated with pembrolizumab, durvalumab or atezolizumab, the TCR of tumor-infiltrating T cells were sequenced from fresh-frozen, paraffinembedded (FFPE) tissue blocks, which led to the identification of highly diverse population (1557 to 
41,435) of TCR clonotypes [29]. Limited by a small sample size, no conclusion could be made in terms of correlation with longer CSS $(R=-0.522, p=0.055)$. In a retrospective cohort study of 29 metastatic urothelial cancer patients treated with atezolizumab, the TCR clonality of peripheral T cells were analyzed [46]. Pretreatment peripheral blood TCR clonality below the median was associated with improved PFS $(p=0.048)$ and OS $(p=0.01)$. A high pretreatment peripheral blood TCR clonality was strongly associated with poor clinical outcome. However, following atezolizumab treatment, expansion of TCR clones in the peripheral blood was associated with durable clinical response $(p=0.01)$. This suggests that low clonality prior to treatment, followed by increased clonality post-treatment is beneficial. These studies, though small in size, suggest that analysis of TCR clonality in blood as well as in the tumor microenvironment (TME) would provide clinical insight to response, and that expansion of TCR clones following treatment is associated with clinical benefit.

\section{Epithelial-Mesenchymal Transition (EMT)}

Equally important as discovering molecular markers to predict clinical benefit is the need to discover markers to predict the potential to do harm. Epithelial-mesenchymal transition (EMT) is the process in which epithelial cells, which normally interacts with the basement membrane via its basal surface, undergo multiple changes, enabling cells to assume a mesenchymal phenotype, which enhances migratory capacity. In this context, EMT has been associated with more aggressive tumor types [53, 54]. One study suggests that EMT presence, determined based on a 200-gene signature expression from The Molecular Signatures Database (MSigDB), may be a potential mechanism of tumor immune evasion during nivolumab treatment of urothelial cancers with high $\mathrm{CD}^{+} \mathrm{T}$ cell infiltration, which are normally immunologically responsive to tumor cells [30]. EMT, as a marker on its own, at high levels is associated with worse ORR (EMT high $13.2 \%$ vs EMT low 23.6\%), PFS (EMT high 1.91 [1.81-2.46] vs EMT low 2.10 [1.87-3.65]) and OS (EMT high 6.57 [4.96-NR] vs EMT low (8.74 [6.05-NR) [30]. EMT has historically been associated with suboptimal response to chemotherapy, progression of disease and development of metastases [53, 54]. This is a longstanding problem that is complicated and poorly understood. Key questions will include whether the mesenchymal state can be reverted to an epithelial state to re-establish an immune-sensitive microenvironment, or otherwise develop strategies to address cancer with mesenchymal properties directly.

\section{Transforming growth factor $\beta$ (TGF $\beta)$}

Mariathason et al, using samples from the IMvigor210 trial which looked at a large cohort of $\mathrm{mBC}$ patients, found that a lack of response to atezolizumab was associated with transforming growth factor $\beta$ (TGF $\beta$ ) signaling in fibroblasts [45]. TGF $\beta$ is particularly associated with a lack of response in patients whose tumors are classified by an "excluded tumor-immune" phenotype, which is defined as the localization of the immune cells, particularly the $\mathrm{CD}^{+} \mathrm{T}$ cells, in the fibroblast and peritumoral stroma, rather than in the tumor parenchyma. Combining anti-TGF $\beta$ and anti-PD-L1 may have the potential to reverse checkpoint resistance, based on early data in murine tumor models [45]. In conclusion, further investigation on the role of TGF $\beta$ will be paramount to inform future strategies to address intrinsic resistance and perhaps acquired resistance to PD-1/PD-L1 inhibitors.

\section{DISCUSSION}

Over the last few years, results from multiple studies have led to accelerated FDA approval of multiple ICIs. Results from these clinical trials led to a shift in $\mathrm{mBC}$ treatment paradigms with the inclusion of ICIs in international guidelines that now recommend atezolizumab and pembrolizumab in the first-line setting for cisplatin-ineligible patients with high PD-L1 score or regardless of PDL1 status in platinum-ineligible patients $[55,56]$. Moreover, in the second-line setting, atezolizumab, pembrolizumab and nivolumab are recommended for $\mathrm{mBC}$ patients with progression during or after platinum-based combination chemotherapy $[55,56]$. Avelumab and durvalumab are recommended for the treatment of locally advanced or metastatic urothelial carcinoma whose disease progressed during or following platinum-containing chemotherapy or within 12 months of neoadjuvant or adjuvant platinumcontaining chemotherapy $[57,58]$.

Along with these encouraging developments, many questions arise since the cost of ICI treatment is significant and the toxicity is not negligible. These questions are driven by the observed heterogeneity of response to ICIs, the risk of pseudoprogression (initial tumor burden increase followed by tumor 
shrinkage) which introduces further challenges to the decision-making process in clinical practice, as well as accelerated disease progression in a small subset of patients (hyperprogression) [23, 59-62]. One important change is the FDA requirement for cisplatin-ineligible patients to have a sufficiently high PD-L1 score before the start of PD-1/PD-L1 inhibitor monotherapy since decreased survival in patients with low PD-L1 expression treated with pembrolizumab or atezolizumab in the first-line setting was shown in KEYNOTE-361 and IMvigor-130 [19-21]. On the other hand, patients who are not eligible for any platinum-based chemotherapy may receive pembrolizumab or atezolizumab regardless of PD-L1 score.

The immune response is a complex system that involves both the tumor and its TME including the complete spectrum of immune cells (e.g. cytotoxic $\mathrm{T}$ cells, suppressive regulatory $\mathrm{T}$ cells, natural killer cells, macrophages). Most studies on ICIs investigated the role of PD-L1 protein expression on either the tumor alone and/or expression on immune cells depending on which PD-L1 antibody was used. Overall, PD-L1 expression correlates with higher response rates, but also a large portion of the patients with a high PD-L1 expression do not respond, whereas some considered PD-L1 low or negative can still respond to treatment. While progress has been made to identify novel predictive biomarkers of response to ICIs, such as TMB, TCR clonality, and IFN $\gamma$ signature, most studies should be considered preliminary due to small cohort size and the lack of independent validation (Fig. 2). The complexity of the tumor microenvironment, and the lack of robust models to study these interactions currently hamper progress. Although the discrepant findings between PD-1/PDL1 ICI studies could be caused by the use of different antibody clones, staining platforms, and scoring systems (Table 1), a recent study indicated a pairwise agreement of $80-90 \%$ between the most frequently used IHC PD-L1 antibodies (22C3, 28-8, SP12 and SP263) [63].

Combining ICI therapies that enhance anti-tumor immunity has been an area of great interest. This is reflected by the number of clinical trials exploring combinations aimed at enhancing response to this relatively new class of anti-cancer drugs, which has soared from a single trial in 2009 to over 1100 in 2017 [64]. Some examples in bladder cancer of active, recruiting trials include a single-arm phase 2 trial looking at neoadjuvant pembrolizumab in combination with gemcitabine and cisplatin

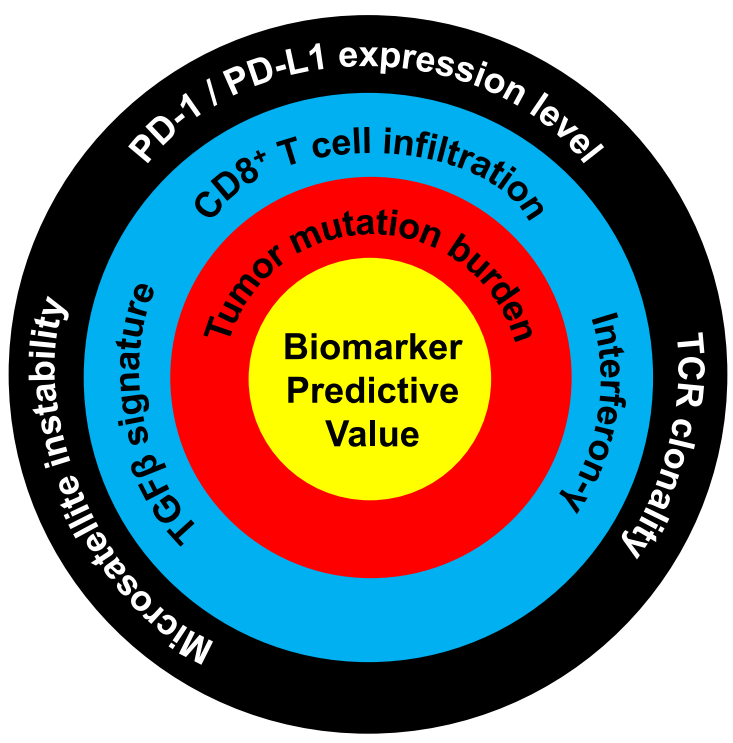

Fig. 2. Ranking of the predictive value of molecular biomarkers for response to PD-1/PD-L1 immune checkpoint blockade. Biomarkers closer to the center are more predictive of response. High predictive value: tumor mutation burden. Moderate predictive value: TGF $\beta$ signature, CD8 T cell infiltration, interferon- $\gamma$. Low predictive value: microsatellite instability, PD-1/PD-L1 expression level, TCR clonality. Predictive level was assessed based on the amount of published data and the quality of these studies, as evaluated and determined in consensus by all authors.

(NCT02690558). The BLASST-1 (Bladder Cancer Signal Seeking Trial) phase 2 study is recruiting MIBC patients with localized disease to explore a combination of nivolumab with gemcitabine and cisplatin in the neoadjuvant setting before a cystectomy (NCT03294304). Other phase 2 trials include combination studies of avelumab with chemoradiation in MIBC patients (NCT03617913) and nivolumab with radiotherapy in cisplatin-ineligible patients (NCT03421652).

In conclusion, we still have not uncovered the true mechanisms leading to effective tumor clearance by ICIs, and no single available biomarker can stratify patient response. As the tumor-related immune response is a multistep process that involves multiple aspects of the immune system, a nomogram combining biomarkers from different steps of the response could provide the solution to this complex interaction. Additionally, more studies are needed to elucidate the molecular genetic underpinnings of the fundamental biological response to ICI treatment. Recently, application of functional genomic approaches has yielded novel insights to such mechanism and has the potential for determining effective drug combinations with 
ICIs, as well as predictive biomarkers for the use of such combinations [65].

\section{ACKNOWLEDGMENTS}

The authors have no acknowledgments.

\section{FUNDING}

The authors report no funding.

\section{CONFLICTS OF INTEREST}

DT reports: Consultant: Machavert, Merck, Urogen; Honoraria: Machavert, Merck, Urogen; licenses/patents: University of Colorado; research support: Medivation/Astellas; stockholder in: Aurora Oncology, PrecisionProfile; other: IOS Press (editorial responsibilities, compensated).The other authors report no conflicts of interest.

\section{REFERENCES}

[1] von der Maase H, Hansen SW, Roberts JT, Dogliotti L, Oliver T, Moore MJ, et al. Gemcitabine and cisplatin versus methotrexate, vinblastine, doxorubicin, and cisplatin in advanced or metastatic bladder cancer: Results of a large, randomized, multinational, multicenter, phase III study. Journal of Clinical Oncology : Official Journal of the American Society of Clinical Oncology. 2000;18(17):3068-77.

[2] Galsky MD, Hahn NM, Rosenberg J, Sonpavde G, Hutson $\mathrm{T}$, Oh WK, et al. A consensus definition of patients with metastatic urothelial carcinoma who are unfit for cisplatin-based chemotherapy. The Lancet Oncology. 2011;12(3):211-4.

[3] Powles T, Eder JP, Fine GD, Braiteh FS, Loriot Y, Cruz C, et al. MPDL3280A (anti-PD-L1) treatment leads to clinical activity in metastatic bladder cancer. Nature. 2014;515(7528):558-62.

[4] Sharma P, Callahan MK, Bono P, Kim J, Spiliopoulou $\mathrm{P}$, Calvo $\mathrm{E}$, et al. Nivolumab monotherapy in recurrent metastatic urothelial carcinoma (CheckMate 032): A multicentre, open-label, two-stage, multi-arm, phase 1/2 trial. The Lancet Oncology. 2016;17(11):1590-8.

[5] Plimack ER, Bellmunt J, Gupta S, Berger R, Chow LQ, Juco J, et al. Safety and activity of pembrolizumab in patients with locally advanced or metastatic urothelial cancer (KEYNOTE-012): A non-randomised, open-label, phase 1b study. The Lancet Oncology. 2017;18(2):212-20.

[6] Massard C, Gordon MS, Sharma S, Rafii S, Wainberg ZA, Luke J, et al. Safety and Efficacy of Durvalumab (MEDI4736), an Anti-Programmed Cell Death Ligand-1 Immune Checkpoint Inhibitor, in Patients With Advanced Urothelial Bladder Cancer. Journal of clinical oncology : Official Journal of the American Society of Clinical Oncology. 2016;34(26):3119-25.

[7] Apolo AB, Infante JR, Balmanoukian A, Patel MR, Wang D, Kelly K, et al. Avelumab, an Anti-Programmed Death-
Ligand 1 Antibody, In Patients With Refractory Metastatic Urothelial Carcinoma: Results From a Multicenter, Phase Ib Study. Journal of Clinical Oncology : Official Journal of the American Society of Clinical Oncology. 2017;35(19):211724.

[8] Cochrane Controlled Register of Trials (CENTRAL): John Wiley \& Sons, Inc.; 2019 Available from: https://www. cochranelibrary.com/central/about-central.

[9] Moher D, Liberati A, Tetzlaff J, Altman DG. Preferred reporting items for systematic reviews and meta-analyses: The PRISMA statement. PLoS medicine. 2009;6(7):e1000097.

[10] Richardson WS, Wilson MC, Nishikawa J, Hayward RS. The well-built clinical question: A key to evidence-based decisions. ACP Journal Club. 1995;123(3):A12-3.

[11] Rosenberg JE, Hoffman-Censits J, Powles T, van der Heijden MS, Balar AV, Necchi A, et al. Atezolizumab in patients with locally advanced and metastatic urothelial carcinoma who have progressed following treatment with platinumbased chemotherapy: A single-arm, multicentre, phase 2 trial. Lancet (London, England). 2016;387(10031):190920.

[12] Balar AV, Galsky MD, Rosenberg JE, Powles T, Petrylak DP, Bellmunt $\mathrm{J}$, et al. Atezolizumab as first-line treatment in cisplatin-ineligible patients with locally advanced and metastatic urothelial carcinoma: A singlearm, multicentre, phase 2 trial. Lancet (London, England). 2017;389(10064):67-76.

[13] Eisenhauer EA, Therasse P, Bogaerts J, Schwartz LH, Sargent D, Ford R, et al. New response evaluation criteria in solid tumours: Revised RECIST guideline (version 1.1). European Journal of Cancer (Oxford, England : 1990). 2009;45(2):228-47.

[14] Powles T, Duran I, van der Heijden MS, Loriot Y, Vogelzang NJ, De Giorgi U, et al. Atezolizumab versus chemotherapy in patients with platinum-treated locally advanced or metastatic urothelial carcinoma (IMvigor211): A multicentre, open-label, phase 3 randomised controlled trial. Lancet (London, England). 2018;391(10122):748-57.

[15] Powles T, Rodriguez-Vida A, Duran I, Crabb SJ, Heijden MSVD, Pous AF, et al. A phase II study investigating the safety and efficacy of neoadjuvant atezolizumab in muscle invasive bladder cancer (ABACUS). Journal of Clinical Oncology. 2018;36(6_suppl).

[16] Bellmunt J, de Wit R, Vaughn DJ, Fradet Y, Lee JL, Fong L, et al. Pembrolizumab as Second-Line Therapy for Advanced Urothelial Carcinoma. The New England Journal of Medicine. 2017;376(11):1015-26.

[17] Bellmunt J, Wit RD, Vaughn DJ, Fradet Y, Lee J-L, Fong L, et al. Two-year follow-up from the phase 3 KEYNOTE045 trial of pembrolizumab (pembro) vs investigator's choice (paclitaxel, docetaxel, or vinflunine) in recurrent, advanced urothelial cancer (UC). Journal of Clinical Oncology. 2018;36(6_suppl):410.

[18] Balar AV, Castellano D, O'Donnell PH, Grivas P, Vuky J, Powles T, et al. First-line pembrolizumab in cisplatinineligible patients with locally advanced and unresectable or metastatic urothelial cancer (KEYNOTE-052): A multicentre, single-arm, phase 2 study. The Lancet Oncology. 2017; 18(11):1483-92.

[19] Keytruda (pembrolizumab) or Tecentriq (atezolizumab): FDA Alerts Health Care Professionals and Investigators: FDA Statement - Decreased Survival in Some Patients in Clinical Trials Associated with Monotherapy: U.S. Food \& Drug Administration; 2018 
[20] Galsky MD, Grande E, Davis ID, Santis MD, Arija JAA, Kikuchi E, et al. IMvigor130: A randomized, phase III study evaluating first-line (1L) atezolizumab (atezo) as monotherapy and in combination with platinum-based chemotherapy (chemo) in patients (pts) with locally advanced or metastatic urothelial carcinoma (mUC). Journal of Clinical Oncology. 2018;36(15_suppl):TPS4589-TPS.

[21] Powles T, Loriot Y, Gschwend JE, Bellmunt J, Geczi L, Vulsteke C, et al. KEYNOTE-361: Phase 3 trial of pembrolizumab \&\#xb1; chemotherapy versus chemotherapy alone in advanced urothelial cancer. European Urology Supplements. 2018;17(2):e1147-e8.

[22] Andrews A. Treating with Checkpoint Inhibitors-Figure $\$ 1$ Million per Patient. American health \& drug benefits. 2015;8(Spec Issue):9.

[23] Kato S, Goodman A, Walavalkar V, Barkauskas DA, Sharabi A, Kurzrock R. Hyperprogressors after Immunotherapy: Analysis of Genomic Alterations Associated with Accelerated Growth Rate. Clinical cancer research : An official journal of the American Association for Cancer Research. 2017;23(15):4242-50.

[24] Postow MA, Sidlow R, Hellmann MD. Immune-Related Adverse Events Associated with Immune Checkpoint Blockade. The New England Journal of Medicine. 2018;378(2):158-68.

[25] Kumar V, Chaudhary N, Garg M, Floudas CS, Soni P, Chandra AB. Current Diagnosis and Management of Immune Related Adverse Events (irAEs) Induced by Immune Checkpoint Inhibitor Therapy. Frontiers in Pharmacology. 2017;8:49.

[26] Sharma P, Callahan MK, Bono P, Kim JW, Spiliopoulou P, Calvo E, et al. Nivolumab monotherapy in metastatic urothelial carcinoma: Longer-term efficacy and safety results from the CheckMate 032 study. Journal of Clinical Oncology. 2018;36(6_suppl):414.

[27] Powles T, O'Donnell PH, Massard C, Arkenau HT, Friedlander TW, Hoimes CJ, et al. Efficacy and Safety of Durvalumab in Locally Advanced or Metastatic Urothelial Carcinoma: Updated Results From a Phase 1/2 Open-label Study. JAMA Oncology. 2017;3(9):e172411.

[28] Patel MR, Ellerton J, Infante JR, Agrawal M, Gordon M, Aljumaily R, et al. Avelumab in metastatic urothelial carcinoma after platinum failure (JAVELIN Solid Tumor): Pooled results from two expansion cohorts of an open-label, phase 1 trial. The Lancet Oncology. 2018;19(1):51-64.

[29] Deng B, Park J-H, Ren L, Yew PY, Kiyotani K, Antic $\mathrm{T}$, et al. CD8 lymphocytes in tumors and nonsynonymous mutational load correlate with prognosis of bladder cancer patients treated with immune checkpoint inhibitors. Cancer Reports. 2018;1(1).

[30] Galsky MD, Wang L, Saci A, Szabo PM, Gong Y, Zhu J. Epithelial-mesenchymal transition (EMT), T cell infiltration, and outcomes with nivolumab (nivo) in urothelial cancer (UC). Annals of Oncology. 2017;28(suppl_5):v295v329.

[31] Gajewski TF, Schreiber H, Fu YX. Innate and adaptive immune cells in the tumor microenvironment. Nature Immunology. 2013;14(10):1014-22.

[32] Templeton AJ, Ace O, McNamara MG, Al-Mubarak M, Vera-Badillo FE, Hermanns T, et al. Prognostic role of platelet to lymphocyte ratio in solid tumors: A systematic review and meta-analysis. Cancer epidemiology, biomarkers \& prevention : A publication of the American Association for Cancer Research, cosponsored by the American Society of Preventive Oncology. 2014;23(7):1204-12.
[33] Guthrie GJ, Charles KA, Roxburgh CS, Horgan PG, McMillan DC, Clarke SJ. The systemic inflammationbased neutrophil-lymphocyte ratio: Experience in patients with cancer. Critical Reviews in Oncology/Hematology. 2013;88(1):218-30.

[34] Zheng Y, Narwal R, Jin C, Baverel PG, Jin X, Gupta A, et al. Population Modeling of Tumor Kinetics and Overall Survival to Identify Prognostic and Predictive Biomarkers of Efficacy for Durvalumab in Patients With Urothelial Carcinoma. Clinical Pharmacology and Therapeutics. 2018;103(4):643-52.

[35] Nakajima C, Uekusa Y, Iwasaki M, Yamaguchi N, Mukai $\mathrm{T}$, Gao $\mathrm{P}$, et al. A role of interferon-gamma (IFN-gamma) in tumor immunity: $\mathrm{T}$ cells with the capacity to reject tumor cells are generated but fail to migrate to tumor sites in IFNgamma-deficient mice. Cancer Research. 2001;61(8):3399405.

[36] Sharma P, Retz M, Siefker-Radtke A, Baron A, Necchi A, Bedke J, et al. Nivolumab in metastatic urothelial carcinoma after platinum therapy (CheckMate 275): A multicentre, single-arm, phase 2 trial. The Lancet Oncology. 2017;18(3):312-22.

[37] Liu MT, Armstrong D, Hamilton TA, Lane TE. Expression of Mig (monokine induced by interferon-gamma) is important in $\mathrm{T}$ lymphocyte recruitment and host defense following viral infection of the central nervous system. Journal of Immunology (Baltimore, Md : 1950). 2001;166(3): 1790-5.

[38] Liu MT, Chen BP, Oertel P, Buchmeier MJ, Armstrong D, Hamilton TA, et al. The T cell chemoattractant IFNinducible protein 10 is essential in host defense against viral-induced neurologic disease. Journal of Immunology (Baltimore, Md : 1950). 2000;165(5):2327-30.

[39] Khan IA, MacLean JA, Lee FS, Casciotti L, DeHaan E, Schwartzman JD, et al. IP-10 is critical for effector T cell trafficking and host survival in Toxoplasma gondii infection. Immunity. 2000;12(5):483-94.

[40] Higgs BW, Morehouse CA, Streicher K, Brohawn PZ, Pilataxi F, Gupta A, et al. Interferon Gamma Messenger RNA Signature in Tumor Biopsies Predicts Outcomes in Patients with Non-Small Cell Lung Carcinoma or Urothelial Cancer Treated with Durvalumab. Clinical Cancer Research : An Official Journal of the American Association for Cancer Research. 2018;24(16):3857-66.

[41] Bais C, Kuziora M, Morehouse C, Higgs BW, Raja R, Lee $\mathrm{Y}$, et al. Biologic and clinical relevance of an IFNG mRNA signature (IFNGS) and PD-L1 protein expression in tumor and immune cells in urothelial cancer (UC) patients (pts) treated with durvalumab (D). Journal of Clinical Oncology. 2017;35(15_suppl):3037.

[42] Ayers M, Lunceford J, Nebozhyn M, Murphy E, Loboda A, Kaufman DR, et al. IFN-gamma-related mRNA profile predicts clinical response to PD-1 blockade. The Journal of Clinical Investigation. 2017;127(8):2930-40.

[43] Lawrence MS, Stojanov P, Polak P, Kryukov GV, Cibulskis $\mathrm{K}$, Sivachenko A, et al. Mutational heterogeneity in cancer and the search for new cancer-associated genes. Nature. 2013;499(7457):214-8.

[44] Rizvi NA, Hellmann MD, Snyder A, Kvistborg P, Makarov V, Havel JJ, et al. Cancer immunology. Mutational landscape determines sensitivity to PD-1 blockade in non-small cell lung cancer. Science (New York, NY). 2015;348(6230):1248.

[45] Mariathasan S, Turley SJ, Nickles D, Castiglioni A, Yuen K, Wang Y, et al. TGFbeta attenuates tumour response to PD- 
L1 blockade by contributing to exclusion of T cells. Nature. 2018;554(7693):544-8.

[46] Snyder A, Nathanson T, Funt SA, Ahuja A, Buros Novik $\mathrm{J}$, Hellmann MD, et al. Contribution of systemic and somatic factors to clinical response and resistance to PD-L1 blockade in urothelial cancer: An exploratory multi-omic analysis. PLoS Medicine. 2017;14(5):e1002309.

[47] Galsky MD, Saci A, Szabo PM, Azrilevich A, Horak C, Lambert A, et al. Impact of tumor mutation burden on nivolumab efficacy in secondline urothelial carcinoma patients: Exploratory analysis of the phase II Checkmate 275 study. Annals of Oncology. 2017;28(suppl_5):v295-v329.

[48] Iyer G, Audenet F, Middha S, Carlo MI, Regazzi AM, Funt $\mathrm{S}$, et al. Mismatch repair (MMR) detection in urothelial carcinoma (UC) and correlation with immune checkpoint blockade (ICB) response. Journal of Clinical Oncology. 2017;35(15_suppl):4511.

[49] Tumeh PC, Harview CL, Yearley JH, Shintaku IP, Taylor EJ, Robert L, et al. PD-1 blockade induces responses by inhibiting adaptive immune resistance. Nature. 2014;515(7528):568-71.

[50] Roh W, Chen PL, Reuben A, Spencer CN, Prieto PA, Miller $\mathrm{JP}$, et al. Integrated molecular analysis of tumor biopsies on sequential CTLA-4 and PD-1 blockade reveals markers of response and resistance. Science Translational Medicine. 2017;9(379).

[51] Page DB, Yuan J, Redmond D, Wen YH, Durack JC, Emerson R, et al. Deep Sequencing of T-cell Receptor DNA as a Biomarker of Clonally Expanded TILs in Breast Cancer after Immunotherapy. Cancer Immunology Research. 2016;4(10):835-44.

[52] Cha E, Klinger M, Hou Y, Cummings C, Ribas A, Faham $\mathrm{M}$, et al. Improved survival with $\mathrm{T}$ cell clonotype stability after anti-CTLA-4 treatment in cancer patients. Science Translational Medicine. 2014;6(238):238ra70.

[53] Smith BN, Bhowmick NA. Role of EMT in Metastasis and Therapy Resistance. Journal of Clinical Medicine. 2016;5(2).

[54] Kalluri R, Weinberg RA. The basics of epithelialmesenchymal transition. The Journal of Clinical Investigation. 2009;119(6):1420-8.

[55] Witjes JA, Bruins M, Compérat E, Cowan NC, Gakis G, Hernández V, et al. Muscle-invasive and Metastatic Bladder Cancer: European Assoication of Urology; 2019 Available from: https://uroweb.org/guideline/bladder-cancer-muscleinvasive-and-metastatic/\#7.

[56] Bladder Cancer NCCN Evidence Blocks. National Comprehensive Cancer Network Clinical Practise Guidelines in Oncology (NCCN Guidelines) 2018; Version 1.2019.
[57] European Association U. European Association of Urology Guidelines. 2018 Edition. Arnhem, The Netherlands: European Association of Urology Guidelines Office; 2018.

[58] Network NCC. Bladder Cancer 2018 [updated July 3, 2018. Version 5.2018:Available from: https://www2.trikobe.org/nccn/guideline/urological/english/bladder.pdf.

[59] Wolchok JD, Hoos A, O'Day S, Weber JS, Hamid O, Lebbe C, et al. Guidelines for the evaluation of immune therapy activity in solid tumors: Immune-related response criteria. Clinical Cancer Research : An Official Journal of the American Association for Cancer Research. 2009;15(23):7412-20.

[60] Ferrara R, Mezquita L, Texier M, Lahmar J, AudigierValette C, Tessonnier L, et al. Hyperprogressive Disease in Patients With Advanced Non-Small Cell Lung Cancer Treated With PD-1/PD-L1 Inhibitors or With Single-Agent Chemotherapy. JAMA Oncology. 2018;4(11):1543-52.

[61] Chiou VL, Burotto M. Pseudoprogression and ImmuneRelated Response in Solid Tumors. Journal of Clinical Oncology : Official Journal of the American Society of Clinical Oncology. 2015;33(31):3541-3.

[62] Champiat S, Dercle L, Ammari S, Massard C, Hollebecque A, Postel-Vinay S, et al. Hyperprogressive Disease Is a New Pattern of Progression in Cancer Patients Treated by Anti-PD-1/PD-L1. Clinical Cancer Research : An Official Journal of the American Association for Cancer Research. 2017;23(8):1920-8.

[63] Rijnders M, van der Veldt AAM, Zuiverloon TCM, Grunberg K, Thunnissen E, de Wit R, et al. PD-L1 Antibody Comparison in Urothelial Carcinoma. European Urology. 2018.

[64] Schmidt C. The benefits of immunotherapy combinations. Nature. 2017;552(7685):S67-s9.

[65] Tu MM, Lee FYF, Jones RT, Kimball AK, Saravia E, Graziano RF, et al. Targeting DDR2 enhances tumor response to anti-PD-1 immunotherapy. Science Advances. 2019;5.

[66] Hahn NM, Powles T, Massard C, Arkenau H-T, Friedlander TW, Hoimes CJ, et al. Updated efficacy and tolerability of durvalumab in locally advanced or metastatic urothelial carcinoma (UC). Journal of Clinical Oncology. 2017;35(15_suppl):4525. 


\section{APPENDIX 1}

Database: Embase Classic+Embase $<1947$ to 2019 January 07>, Ovid MEDLINE(R) ALL <1946 to January 07, 2019>, EBM Reviews - Cochrane Central Register of Controlled Trials <December 2018>

Search Strategy:

1 Urinary Bladder Neoplasms/sc [Secondary] (591)

2 Carcinoma, Transitional Cell/sc [Secondary] (1397)

3 Urinary Bladder Neoplasms/ or Carcinoma, Transitional Cell/ (76902)

4 ((bladder or urothelial) adj3 (cancer* or neoplasm* or tumor* or tumour* or carcinoma*)).tw. (133033)

5 ((bladder or urothelial) and (cancer* or neoplasm* or tumor* or tumour* or carcinoma*)).kf. (9487)

63 or 4 or 5 (158803)

7 exp Neoplasm Metastasis/ (797411)

8 metast*.tw,kw. (1167908)

97 or 8 (1390214)

106 and $9(28881)$

111 or 2 or 10 (29336)

12 atezolizumab.mp. (3325)

13 durvalumab.mp. (2245)

14 avelumab.mp. (1399)

15 BMS-936559.mp. (392)

16 nivolumab.mp. (13963)

17 pembrolizumab.mp. (11027)

18 (checkpoint block* or check point block* or checkpoint inhibitor* or check point inhibitor*).tw,kw. (20623)

19 PD-L1.tw,kw. (22737)

20 PD-1.tw,kw. (29759)

21 (immune checkpoint or immune check point).tw,kw. (18138)

22 (CTLA-4 or CTLA4).tw,kw. (22889)

23 CTLA-4 Antigen/ai [Antagonists \& Inhibitors] (691)

24 Programmed Cell Death 1 Receptor/ai [Antagonists \& Inhibitors] (1372)

25 or/12-24 (75074)

2611 and $25(1297)$

27 exp animals/ not humans/ (16935118)

2826 not 27 (902)

2928 use medall (316) Medline

30 exp bladder cancer/ (116923)

31 transitional cell carcinoma/ (43663)

3230 or 31 (136611)
33 exp metastasis/ (797411)

3432 and 33 (16760)

35 (metast* adj5 ((bladder or urothelial) adj3 (cancer* or neoplasm* or tumor* or tumour* or carcinoma*))).tw. (8104)

36 bladder metastasis/ (1197)

3734 or 35 or 36 (21293)

38 antineoplastic monoclonal antibody/ or atezolizumab/ or avelumab/ or bms $936559 /$ or durvalumab/ or nivolumab/ or pembrolizumab/ (15335)

39 (atezolizumab or avelumab or bms 936559 or durvalumab or nivolumab or pembrolizumab).tw. (14518)

40 (checkpoint block* or check point block* or checkpoint inhibitor* or check point inhibitor*).tw. (19604)

41 ((ctla-4 or pd-1 or pd-11) adj4 (antibod* or inhibitor*)).tw. (15052)

42 *programmed death 1 ligand 1/ (5291)

43 or $/ 38-42$ (43827)

4437 and 43 (1115)

45 (exp animal/ or nonhuman/ or animal experiment/) not exp human/ (11408775)

4644 not 45 (1095)

4746 use emczd (799) Embase

48 Urinary Bladder Neoplasms/sc [Secondary] (591)

49 Carcinoma, Transitional Cell/sc [Secondary] (1397)

50 Urinary Bladder Neoplasms/ or Carcinoma, Transitional Cell/ (76902)

51 ((bladder or urothelial) adj3 (cancer* or neoplasm* or tumor* or tumour* or carcinoma*)).tw. (133033)

52 (bladder cancer or bladder neoplasms* or bladder tumor* or bladder tumour*).kw. (21939)

5350 or 51 or 52 (159545)

54 exp Neoplasm Metastasis/ (797411)

55 metast*.tw,kw. (1167908)

5654 or 55 (1390214)

5753 and 56 (29009)

5848 or 49 or 57 (29464)

59 atezolizumab.mp. (3325)

60 durvalumab.mp. (2245)

61 avelumab.mp. (1399)

62 BMS-936559.mp. (392)

63 nivolumab.mp. (13963)

64 pembrolizumab.mp. (11027)

65 (checkpoint block* or check point block* or checkpoint inhibitor* or check point inhibitor*).tw,kw. (20623) 
66 PD-L1.tw,kw. (22737)

67 PD-1.tw,kw. (29759)

68 (immune checkpoint or immune check point).tw,kw. (18138)

69 (CTLA-4 or CTLA4).tw,kw. (22889)

70 CTLA-4 Antigen/ai [Antagonists \& Inhibitors] (691)

71 Programmed Cell Death 1 Receptor/ai [Antagonists \& Inhibitors] (1372)
72 or/59-71 (75074)

7358 and $72(1302)$

7473 use cctr (122)

7529 or 47 or 74 (1237) Cochrane

76 remove duplicates from 75 (967)

7776 use medall (313) Medline

7876 use emczd (566) Embase

7976 use cctr (88) Cochrane 